\title{
First-principles Studies for the Hydrogen Doping Effects on Iron-based Superconductors
}

\author{
Hiroki Nakamura ${ }^{1,2,3, *}$ and Masahiko Machida ${ }^{1,2,3, \oplus}$ \\ ${ }^{1}$ CCSE, Japan Atomic Energy Agency, , 6-9-3 Higashi-Ueno, Taito-ku Tokyo 110-0015, Japan \\ ${ }^{2}$ CREST (JST), 4-1-8 Honcho, Kawaguchi, Saitama 332-0012, Japan \\ ${ }^{3}$ JST, Transformative Research-Project on Iron Pnictides (TRIP), Chiyoda, Tokyo 102-0075, Japan
}

(Dated: July 3, 2018)

\begin{abstract}
We study hydrogen doping effects in an iron-based superconductor $\mathrm{LaFeAsO}_{1-y}$ by using the first-principles calculation and explore the reason why the superconducting transition temperature is remarkably enhanced by the hydrogen doping. The present calculations reveal that a hydrogen cation stably locating close to an iron atom attracts a negatively-charged FeAs layer and results in structural distortion favorable for further high temperature transition. In fact, the lattice constant $a$ averaged over the employed supercell shrinks and then the averaged As-Fe-As angle $\alpha$ approaches $109.74^{\circ}$ with increasing the hydrogen doping amount. Moreover, the calculations clarify electron doping effects of the solute hydrogen and resultant Fermi-level shift. These insights are useful for design of high transition-temperature iron-based superconductors.
\end{abstract}

PACS numbers: 74.25.Jb, 74.70.-b, 71.15.Mb

Since the discovery of the high-temperature superconductivity in $\mathrm{LaFeAsO}_{1-x} \mathrm{~F}_{x}$ 1], iron-based superconductors have drawn wide attention due to not only their hightemperature transition but also peculiar superconducting properties. According to the accumulated insights, most of iron-based superconductors require chemical doping like substitution as $\mathrm{LaFeAsO}_{1-x} \mathrm{~F}_{x}$ or deficiency as $\mathrm{LaFeAsO}_{1-y}$ for their optimal high- $T_{\mathrm{c}}$ superconductivity [2]. In addition, most of their mother compounds exhibit the stripe-type antiferromagnetic ordering just after the orthorhombic transition on cooling the temperature. There are several similarities with cuprate high- $T_{\mathrm{c}}$ superconductors but some original particularities. Therefore, more systematic research is now still in great demand.

Recently, Miyazawa et al., 3] reported a fascinating fact that the transition temperature $T_{\mathrm{c}}$ of $\mathrm{LaFeAsO}_{1-y}$ is enhanced from $28 \mathrm{~K}$ to $38 \mathrm{~K}$ by doping the hydrogen in its high-pressure synthesis. It surprisingly corresponds to more than $30 \%$ of $T_{\mathrm{c}}$ enhancement, which is unlikely in other superconductors. This new synthesis scheme is quite different from the previous chemical doping such as substitutions and deficiencies, because the enhanced $T_{\mathrm{c}}$ is much beyond the optimum reach of so-called La-1111 compounds. Therefore, the hydrogen doping effect should be an important key to the superconducting mechanism and $T_{\mathrm{c}}$ enhancement in iron-based superconductors. In this paper, we study the hydrogen doping effects through systematic first-principles calculations. The highlights of this paper are to clarify at which the doped hydrogen atom prefers to stay inside the crystal unit and how the doped hydrogen atoms deform the crystalline structure and resultant electronic one. Most of the calculated results are consistent with measurement data, while they contain novel insights never experimentally accessible.

Before starting the calculation study of the hydrogen doping effects, there are a few questions to narrow the point. The first one is whether the hydrogen atoms really keep their positions inside the crystal. The nuclear magnetic resonance measurement on the hydrogen $(\mathrm{H}-$ NMR) confirmed the existence of doped hydrogen atoms [3, 5] not at any surfaces and boundaries but inside the crystal. This indicates that there are stable locations inside the crystal for the hydrogen atom. The next one is internal locations of the doped hydrogen atoms. One naively expects that doped $\mathrm{H}$ atoms fills $\mathrm{O}$ vacancies. Contrary to this conjecture, Fe-NMR verified that the hydrogen atoms partly locate close to Fe atoms [5]. However, their exact locations have not been known yet because of its technical difficulties. Then, we explore the exact position at which the hydrogen atom stably locates around Fe atom by first-principles calculations. Once one solves the above problem, the final goal is to clarify how the hydrogen atom raises $T_{\mathrm{c}}$. In this paper, we reveal some important roles of the doped hydrogen atoms. The first one is that the hydrogen atoms tend to shrink lattice constants like the replacement of Ln element in LnO blocking layer of 1111 compounds. The superconducting transition temperature is known to be quite sensitive to the variation of the lattice constants. Moreover, it is well-known that the As-Fe-As angle, $\alpha$ and the height of an As atom from an Fe-plane, $h_{A s}$ are closely correlated with $T_{\mathrm{c}}[6,7]$. In fact, we find that the doped hydrogen distorts the crystal structure more suitable for high- $T_{\mathrm{c}}$ superconductivity. The second one is whether the hydrogen doping supplies additional carriers. The calculations reveal that the hydrogen provides electron and the Fermi surfaces change their shapes in a large doping level.

Let us briefly show the present calculation techniques. We examine hydrogen-doping effects in iron-based superconductors based on the density functional theory (DFT). The calculation package employed throughout 
this paper is VASP [8], which adopts PAW method [10] and GGA [9] exchange-correlation energy. In order to examine how the doped hydrogen deforms the crystal structure, we prepare $2 \times 2 \times 1$ supercell of $(\mathrm{LaFeAsO})_{8}$ as shown in Fig. 1(a). If one puts a hydrogen atom into the supercell, the chemical formula is given by $\mathrm{LaFeAsOH}_{x}$, in which $x=0.125$. Accordingly, $x=0.25$ when inserting two $\mathrm{H}$ atoms. Thus, we can control the doping level $x$, although the variation range is quantized. In order to find relaxed optimal positions for the hydrogen atoms inside the supercell, we optimize the crystal structure of $\mathrm{LaFeAsOH}_{x}$, so that the forces on all atoms become less than $0.01 \mathrm{eV} / \AA$. The parameters set in the DFT calculations are given as follows. $k$-points are taken $4 \times 4 \times 4$, and the self-consistent loops are repeated until the energy deviation becomes less than $10^{-5} \mathrm{eV}$ with the cut-off energy being $500 \mathrm{eV}$.

Now, let us present the calculation results. First, we explore stable positions for a hydrogen atom inside LaFeAsO crystal unit. We initially prepare a few tens configurations, in which an $\mathrm{H}$ atom is randomly distributed inside the LaFeAsO unit cell, and optimize their structures. Then, the doped hydrogen atom finds out a stable location. As a result, we find two typical locations for an additive $\mathrm{H}$ atom. The first type is around an alternately vacant As position which is mirror symmetric with a non-vacant As across the Fe-plane. According to the calculation result, the exact stable location slightly shifts from the vacant As position as shown in Fig. 1(b). The second one is just below a La atom alternately symmetric across the $\mathrm{O}$ plane as shown in Fig. 1(c). The former case is found to be more stable by comparing their total energies. This result actually agrees with Fe-NMR measurements [5]. However, the calculated energy difference is $50 \mathrm{meV}$ per $\mathrm{LaFeAsOH}_{0.125}$ and the hydrogen atom may partially occupy the second position as a quasistable location.

Here, we turn to $\mathrm{O}$ vacancies. As mentioned above, $\mathrm{H}$ atoms are expected to enter $\mathrm{O}$ vacancies. In order to examine this, we compare the total energies of the following two types of $(\mathrm{LaFeAs})_{8} \mathrm{O}_{7} \mathrm{H}$ structures. A configuration is that a $\mathrm{H}$ atom fills the $\mathrm{O}$ vacancy, and the other one is that it locates near an $\mathrm{Fe}$ atom without filling the $\mathrm{O}$ vacancy. As a result, more stable configuration is found to be the former one. Therefore, we conclude that $\mathrm{H}$ atoms first tend to fill $\mathrm{O}$ vacancies. In this case, the $\mathrm{H}$ doping corresponds to just a substitution like $\mathrm{LaFeAsO}_{1-x} \mathrm{~F}_{x}$, and it is found to be not relevant to more than $30 \%$ $T_{\mathrm{c}}$ enhancement. In fact, the Fe-NMR result [5] insists that $\mathrm{H}$ atoms exist near $\mathrm{Fe}$ atoms. This indicates that an excess portion of the doped $\mathrm{H}$ atoms located in FeAs layers. Hereafter, we focus on the $\mathrm{H}$ locations near $\mathrm{Fe}$ atoms as a new mechanism of $T_{\mathrm{c}}$ enhancement.

Let us concentrate on the location inside FeAs layers. As shown in Fig. 1(b), we find out that the most stable position shifts to $(0.07,0.07)$ in the inner atomic coordi-
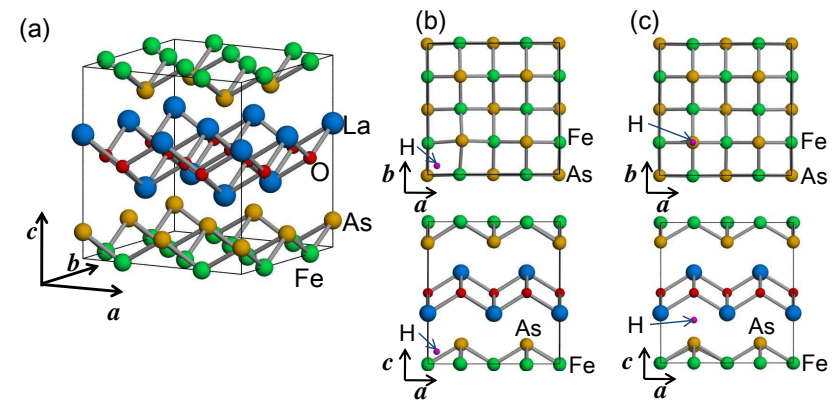

FIG. 1: (a) $2 \times 2 \times 1$ supercell of LaFeAsO. (b) Most stable structure of $(\mathrm{LaFeAsO})_{8} \mathrm{H}$, in which a $\mathrm{H}$ atom exists in the FeAs layer. (c) Metastable structure of $(\mathrm{LaFeAsO})_{8} \mathrm{H}$ which contains a $\mathrm{H}$ atom around the $\mathrm{LaO}$ layer.

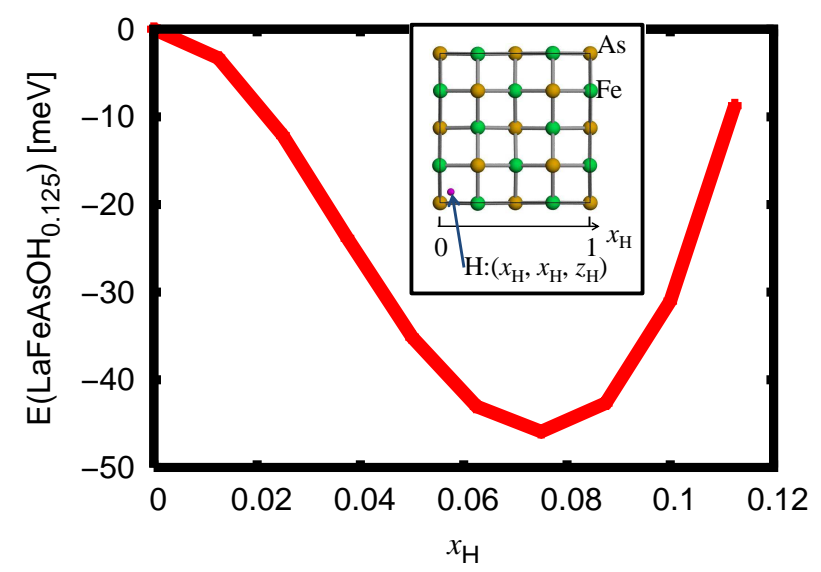

FIG. 2: Total energy per $\mathrm{LaFeAsOH}_{0} .125$ as a function of inner atomic coordinates of the $\mathrm{H}$ atom in $(\mathrm{LaFeAsO})_{8}$. Inset shows the position of the $\mathrm{H}$ atom. Inner atomic coordinate $z_{\mathrm{H}}$, which is along $c$-axis, is optimized.

nate from $(0,0)$ corresponding to just above an Fe atom. We carefully trace a variation of the total energy with respect to the position shift as $\left(x_{\mathrm{H}}, x_{\mathrm{H}}, z_{\mathrm{H}}\right)$, in which once $x_{\mathrm{H}}$ is fixed $z_{\mathrm{H}}$ is optimized. Figure 2 shows the total energy variation as a function of $x_{\mathrm{H}}$. At $x_{\mathrm{H}}=0$, the total energy counterintuitively shows maximum. The present calculation reveals the reason as follows. The position $x_{\mathrm{H}}=0$ is actually unstable due to the repulsion from the nearest $\mathrm{La}^{3+}$ cation for the $\mathrm{H}^{+}$one. Then, it shifts to avoid the so-called Coulomb-repulsion pressure from the $\mathrm{La}^{3+}$ cation. This result obviously indicates that Ln cation in 1111 crystals has an important influence on FeAs layer. The Ln cation and FeAs layer keep exquisite balance. On the other hand, we notice in Fig. 2 that the energy difference in the energy valley is not so large that the hydrogen atom can largely vibrate around the stable position. Such a localized dynamical effect is beyond the present study.

Next, we examine the hydrogen-doping amount dependence. Since we now know the most stable location inside 


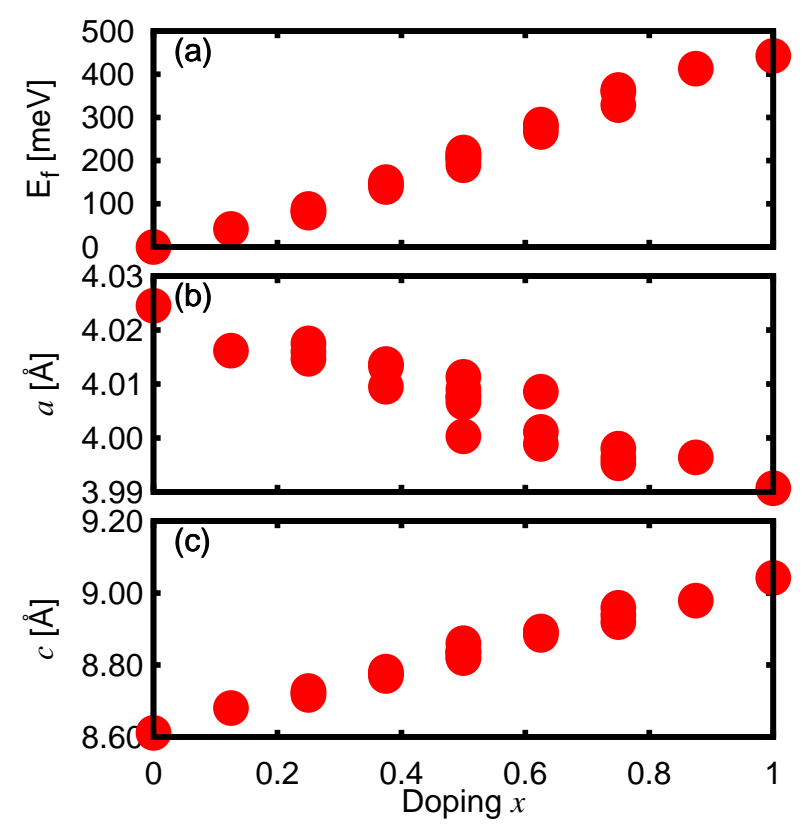

FIG. 3: $E_{\mathrm{f}}(x)$ (see Eq. (1)) (a), lattice constants $a(\mathrm{~b})$ and $c$ (c) as a function of doping $x$.

the crystal unit, as initial configurations we put $\mathrm{H}$ atoms on the positions as shown in Fig प(b). There are 1 to 8 As atoms inside the present super-cell. This indicates that there are also 8 possible initial locations for an $\mathrm{H}$ atom. Then, the number of possible configurations are totally $2^{8}=256$. Among them, several configurations are symmetrically equivalent to others, and the initial sets can be then reduced to 22 patterns, among which the number of doped hydrogen atoms is ranged from 1 to 8. We calculate stable structures and total energies on these all 22 patterns. Figure 3(a) shows an energy $E_{\mathrm{f}}(x)$ as a function of the doping level $x$, which is obtained by dividing the number of $\mathrm{H}$ atoms in the cell by 8 . The energy $E_{\mathrm{f}}(x)$ is defined as

$$
E_{\mathrm{f}}(x)=E\left(\mathrm{LaFeAsOH}_{x}\right)-E(\mathrm{LaFeAsO})-\frac{x}{2} E\left(\mathrm{H}_{2}\right),
$$

where $E(\cdots)$ stands for the total energy of the correspondent chemical formula. As seen in Fig. 3(a), $E_{\mathrm{f}}(x)$ is positive and increases with $x$. One finds that all the present possible doping is energetically unstable compared to non-doped LaFeAsO. This is related to a fact that high pressure synthesis is needed for doping. The doping $x$ variation of the lattice constants $a$ and $c$ are shown in Figs. 3(b) and (c), respectively. From both the figures, one finds that $a$ decreases with increasing $x$. The observed $a$ of $\mathrm{H}$-doped $\mathrm{LaFeAsO}_{1-y}$ is 3.9938 $\AA[3]$, which is distinctly smaller than that of the nondoped $\mathrm{LaFeAsO}_{1-y}(4.0257 \AA)[4]$. This fact is consistent with our calculations. On the other hand, the observed $c$ counterintuitively decreases from $8.7190 \AA[4]$ to 8.6898 $\AA[3]$ by $\mathrm{H}$ doping, although the lattice constant $c$ in-

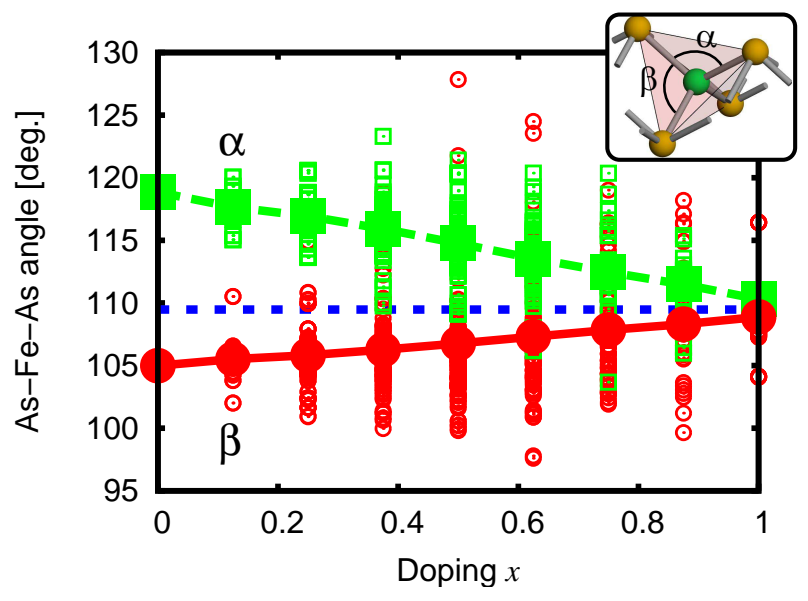

FIG. 4: As-Fe-As angles as a function of doping $x$. Small squares and circles stand for $\alpha$ and $\beta$ angles, respectively, which are depicted in the inset. Large squares and circles are the average of $\alpha$ and $\beta$, respectively. Horizontal line denotes the regular tetrahedron angle, $109.47^{\circ}$.

creases with $x$ in the present calculation. The present tendency contradicts the measurement data. We suggest a reason on the opposite tendency as follows. It is wellknown that the hydrogen can easily diffuse in various compounds because the energy barrier for the diffusion is not so high as seen in Fig,2. This means that any hydrogens do not always occupy the energy minimum point. For instance, if a hydrogen atom partially occupies the second energy minimum location as shown in Fig 1 (c), then $c$-axis constant may rather decreases, because the neighbor is negatively-charged $\mathrm{O}^{2-}$. Although such statistical analyses on various configurations including the diffusion effects are beyond the present work, the $c$-axis shrinkage clearly indicates that doped hydrogen atoms partly stay at the positions close to the anions.

Figure 4 is the highlight of the present paper. Lee et al., [6] pointed out that $T_{\mathrm{c}}$ is closely correlated to the As-Fe-As angle, $\alpha$ (see the inset of Fig. 4, where $\beta$ is noted to be also another equivalent index). $T_{\mathrm{c}}$ takes the maximum value when $\alpha(\beta)=109.47^{\circ}$, at which $\mathrm{FeAs}_{4}$ tetrahedron becomes a perfectly regular one. As seen in Fig. 4, the averaged $\alpha(\beta)$ clearly approaches to the optimal value with increasing the hydrogen doping ratio $x$. The reason is given as follows. The hydrogen ion (cation) attracts negatively-charged FeAs layer, and then the inplane lattice constants decrease. Consequently, the As height increases and the angle $\alpha(\beta)$ decreases(increases).

The next issue is an effect of the hydrogen doping on the electronic band structure. We display band dispersions of $\mathrm{H}$-doped $\mathrm{LaFeAsO}$ in Fig. 5. It is noted that the current Brillouin zone (BZ) is folded to a quarter due to the use of $2 \times 2$ supercell. In the conventional unit cell, the iron-based superconductors generally have hole pockets around the $\Gamma$ point (the center of BZ) and electron pockets around $M$ point (the corner of $\mathrm{BZ}$ ). In the present 

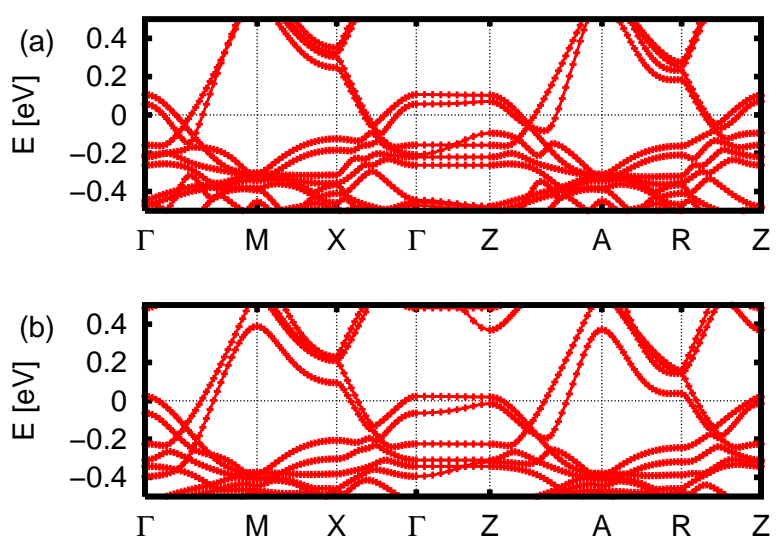

FIG. 5: Band structure of $\mathrm{LaFeAsOH}_{x}$ of (a) $x=0.125$ (b) $x=0.25$. The Fermi energy is set to zero.

zone, $M$ point moves to $\Gamma$ one by the zone folding, and both the pockets almost overlap around $\Gamma$ one. Actually, both the pockets crosses in the case at $x=0.125$ as displayed in Fig. $5(a)$, i.e., the Fermi surfaces between the hole and electron pockets are well nested. Meanwhile, the hole pocket almost disappears at $x=0.25$ as shown in Fig. 5(b). The reason is that the doped hydrogen atom becomes a cation and supplies an electron. Consequently, the Fermi level lifts up and the hole pocket disappears beneath the Fermi level. This indicates that too much hydrogen doping drastically changes the electronic structure around the Fermi level and eventually diminishes the high-temperature superconductivity.

Finally, we discuss the hydrogen doping effects together with various knowledge on hydrogen additives in solid states. First, we stress that the doped hydrogen shrinks all the lattice constants although extra element additives normally expand lattice constants. One of the origin is that the hydrogen cation locating stably close to Fe atom attracts negatively-charged FeAs layer. Thus, the mother compound LaFeAsO crystal is found to be rather loosely packed in contrast to very low solubility of the hydrogen in pure Fe crystal. This fact is deeply relevant to the large pressure and substitution effects in 1111 compounds. From this discussion, it is expected that the hydrogen doping effect on Sm-, Gd- and Nd1111 compounds leads to further $T_{\mathrm{c}}$ enhancement. Unfortunately, such enhancement was not reported, e.g., in $\mathrm{H}$-doped $\mathrm{SmFeAsO}_{1-y}$ [11, 12]. However, in their compounds, it is noteworthy that the hydrogen is also soluble, because their FeAs structures and associated electronic ones are still controllable. Second, we point out that the doped hydrogen supplies an electron. Namely, the hydrogen doping lifts up the Fermi level and the excess doping loses the nesting situation which is regarded as an electronic-structure stage for high- $T_{\mathrm{c}}$ superconductivity. This means that there is the best hydrogen doping ra- tio while further $T_{\mathrm{c}}$ enhancement can be expected if one controls the carrier number by different ways from the hydrogen doping, because the angle $\alpha$ monotonically approaches the best angle with the doping amount. Third, we would like to mention that the local distortion generated by the doped hydrogen considerably varies inside the supercell unit and just the averaged distortion is preferable for the transition temperature enhancement as shown in Fig. 4. Accordingly, the emergent superconductivity seems to reflect not local atomic-scale but widely averaged distortions and resultant electronicstructure changes. Since high diffusivity of the hydrogen is largely suppressed in a low temperature range as the superconducting transition, it is a big mystery that the interstitial hydrogen close to Fe seems not to work as a pair breaking potential.

We have investigated the optimized crystal structure of the hydrogen doped $\mathrm{LaFeAsOH}_{x}$ by using the density functional theory. The calculation results indicate that the hydrogen atom prefers to stay close to Fe atom. In the case, the supercell calculations revealed that the averaged lattice constant $a$ shrinks with increasing the doping amount, which is consistent with the experimental results. Meanwhile, the lattice constant $c$ expands within the present calculations, although it also shrinks in experiments. Therefore, it is considered that the hydrogen atoms locates at not only the most stable point but also other minimum ones. The averaged structural distortions coincide with the correlation tendency of $T_{\mathrm{c}}$ on the crystal structure. As a typical example, we observed that the As-Fe-As angle $\alpha$ approaches closer to $109.47^{\circ}$ by increasing the amount of the doped hydrogen. However, the doping shifts up the Fermi level, because the hydrogen also has the carrier doping effect. In conclusion, the present first-principles studies clarified some fundamental aspects in the hydrogen doping beyond the findings obtained by experiments. We believe that their insights are essential to materials design for further high$T_{\mathrm{c}}$ superconductivity.

* nakamura.hiroki@jaea.go.jp

$\dagger$ machida.masahiko@jaea.go.jp

[1] Y. Kamihara et al., J. Am. Chem. Soc. 130, 3296 (2008).

[2] See e.g., K. Ishida, Y. Nakai, H. Hosono, J. Phys. Soc. Jpn. 78, 062001 (2009).

[3] K. Miyazawa et al., Appl. Phys. Lett. 96, 072514 (2010).

[4] K. Miyazawa et al., J. Phys. Soc. Jpn. 78, 034712 (2009) ; see also references therein.

[5] H. Yamashita et al., J. Phys. Soc. Jpn. 79, 103703 (2010).

[6] C.-H. Lee et al., J. Phys. Soc. Jpn. 77, 083704 (2008).

[7] Y. Mizuguchi, Y. Takano J. Phys. Soc. Jpn. 79, 102001 (2010).

[8] G. Kresse, J. Hafner, Phys. Rev. B 47, RC558 (1993); G. Kresse, J. Furthmüller, Phys. Rev. B 54, 11169 (1996).

[9] J. P. Perdew, K. Burke, M. Ernzerhof, Phys. Rev. Lett. 
77, 3865 (1996)

[10] P. E. Blöchl, Phys. Rev. B 50, 17953 (1994); G. Kresse and D. Joubert, Phys. Rev. B 59, 1758 (1999).
[11] P.M. Shirage et al., Phys. Rev. Lett. 105, 037004 (2010).

[12] T. Hanna et al., arXiv:1103.1177. 